\title{
A MODIFIED RIEMANN ZETA DISTRIBUTION IN THE CRITICAL STRIP
}

\author{
TAKASHI NAKAMURA
}

(Communicated by Mark M. Meerschaert)

\begin{abstract}
Let $\sigma, t \in \mathbb{R}, s=\sigma+$ it and $\zeta(s)$ be the Riemann zeta function. Put $f_{\sigma}(t):=\zeta(\sigma-\mathrm{i} t) /(\sigma-\mathrm{i} t)$ and $F_{\sigma}(t):=f_{\sigma}(t) / f_{\sigma}(0)$. We show that $F_{\sigma}(t)$ is a characteristic function of a probability measure for any $0<\sigma \neq 1$ by giving the probability density function. By using this fact, we show that for any $C \in \mathbb{C}$ satisfying $|C|>10$ and $-19 / 2 \leq \Re C \leq 17 / 2$, the function $\zeta(s)+C s$ does not vanish in the half-plane $\sigma>1 / 18$. Moreover, we prove that $F_{\sigma}(t)$ is an infinitely divisible characteristic function for any $\sigma>1$. Furthermore, we show that the Riemann hypothesis is true if each $F_{\sigma}(t)$ is an infinitely divisible characteristic function for each $1 / 2<\sigma<1$.
\end{abstract}

\section{INTRODUCTION AND MAIN RESULTS}

The Riemann zeta function $\zeta(s)$ is a function of a complex variable $s=\sigma+\mathrm{i}$, for $\sigma>1$ given by

$$
\zeta(s):=\sum_{n=1}^{\infty} \frac{1}{n^{s}}=\prod_{p}\left(1-\frac{1}{p^{s}}\right)^{-1},
$$

where the letter $p$ is a prime number, and the product of $\prod_{p}$ is taken over all primes. The series is called the Dirichlet series and the product is called the Euler product. The Dirichlet series and the Euler product of $\zeta(s)$ converges absolutely in the half-plane $\sigma>1$ and uniformly in each compact subset of this half-plane. It is known that the Riemann zeta function is a meromorphic function on the whole complex plane, which is holomorphic everywhere except for a simple pole at $s=1$ with residue 1 (see for example [2, p. 35]).

Let $\mu$ be a probability measure on $\mathbb{R}$, namely, $\int_{-\infty}^{\infty} \mu(d y)=1$. For $z \in \mathbb{R}$ the characteristic function $\widehat{\mu}(z)$ of $\mu$ is defined by $\widehat{\mu}(z):=\int_{-\infty}^{\infty} e^{\mathrm{i} z y} \mu(d y)$. For instance, the distribution concentrated at $x \in \mathbb{R}$ is the $\delta$-distribution at $x$ and denoted by $\delta_{x}$, and its characteristic function is given by $e^{\mathrm{i} z x}$ (see [17, Section 2]).

Put $Z_{\sigma}(t):=\zeta(\sigma+\mathrm{i} t) / \zeta(\sigma), t \in \mathbb{R}$, then $Z_{\sigma}(t)$ is known to be a characteristic function when $\sigma>1$ (see [10], [5, p. 75] and [14, Corollary 1]).

Received by the editors April 9, 2013 and, in revised form, May 24, 2013 and June 3, 2013. 2010 Mathematics Subject Classification. Primary 60E10, 11M06; Secondary 60E07, 11M26.

Key words and phrases. Characteristic function, infinite divisibility, zeros of zeta functions.

The author would like to thank the referees for their constructive and helpful comments and suggestions on the manuscript. 
Proposition 1.1. For $\sigma>1$, the measure

$$
\mu_{\sigma}(d y):=\frac{1}{\zeta(\sigma)} \sum_{n=1}^{\infty} \frac{1}{n^{\sigma}} \delta_{-\log n}(d y)
$$

is a probability measure, with characteristic function $Z_{\sigma}(t)=\int_{-\infty}^{\infty} e^{\mathrm{i} t y} \mu_{\sigma}(d y)$.

Moreover $\mu_{\sigma}$ is an infinitely divisible distribution and its Lévy measure (see Section 3) can be given as follows:

$$
\begin{aligned}
& Z_{\sigma}(t)=\exp \left[\int_{0}^{\infty}\left(e^{-\mathrm{i} t x}-1\right) N_{\sigma}(d x)\right], \\
& N_{\sigma}(d x):=\sum_{p} \sum_{r=1}^{\infty} \frac{p^{-r \sigma}}{r} \delta_{r \log p}(d x) .
\end{aligned}
$$

The distribution $\mu_{\sigma}$ on $\mathbb{R}$ is said to be a Riemann zeta distribution with parameter $\sigma$. Recently, Lin and $\mathrm{Hu}$ [14, and Gut 6] investigated the Riemann zeta distribution only in the region of absolute convergence $\sigma>1$. On the other hand, Aoyama and Nakamura [1, Remark 1.13] showed that $Z_{\sigma}(t)$ is not a characteristic function for any $1 / 2 \leq \sigma \leq 1$.

There are some other papers that treat functions related to the Riemann zeta function in probabilistic view. Biane, Pitman and Yor [4] reviewed known results about the probabilistic interpretations of $s(s-1) \pi^{-s / 2} \Gamma(s / 2) \zeta(s)$, where $\Gamma(s)$ is the Gamma function. Lagarias and Rains [11] considered $\pi^{-s / 2} \Gamma(s / 2) \zeta(s)$ and its generalizations and gave results related to infinite divisibility (see Section 3).

In the present paper, we give a modified Riemann zeta distribution in the critical strip $0<\sigma<1$ (despite [1, Remark 1.13] mentioned above) in Theorem 1.2 and consider its application to analytic number theory in Theorem 1.3. Put

$$
f_{\sigma}(t):=\frac{\zeta(\sigma-\mathrm{i} t)}{\sigma-\mathrm{i} t}, \quad F_{\sigma}(t):=\frac{f_{\sigma}(t)}{f_{\sigma}(0)}, \quad 0<\sigma \neq 1 .
$$

By the definitions of $Z_{\sigma}(t)$ and $F_{\sigma}(t)$, we have

$$
F_{\sigma}(t)=\frac{\zeta(\sigma-\mathrm{i} t)}{\zeta(\sigma)} \frac{\sigma}{\sigma-\mathrm{i} t}=Z_{\sigma}(-t) \frac{\sigma}{\sigma-\mathrm{i} t} .
$$

Note that $\sigma /(\sigma-\mathrm{i} t)$ is the characteristic function of the exponential distribution with parameter $\sigma>0$ defined by $\mu(B):=\sigma \int_{B \cap(0, \infty)} e^{-\sigma y} d y$, where $B$ is a Borel set on $\mathbb{R}$, namely, $\sigma /(\sigma-\mathrm{i} t)=\sigma \int_{0}^{\infty} e^{\mathrm{i} t y} e^{-\sigma y} d y$ (see for instance [17, Example 2.14]).

Theorem 1.2. The function $F_{\sigma}(t)$ is a characteristic function of a probability measure for all $\sigma>0$ except for $\sigma=1$. Moreover the associated probability measure is absolutely continuous with density function $P_{\sigma}(y)$ given as follows:

$$
P_{\sigma}(y):= \begin{cases}\frac{\sigma}{\zeta(\sigma)} \frac{\left[e^{y}\right]}{e^{y \sigma}} & \sigma>1, \\ \frac{\sigma}{\zeta(\sigma)} \frac{\left[e^{y}\right]-e^{y}}{e^{y \sigma}} & 0<\sigma<1 .\end{cases}
$$

From the point of view of the Fourier transform, one has $F_{\sigma}(t)=\int_{-\infty}^{\infty} e^{\mathrm{i} t y} P_{\sigma}(y) d y$. This formula should be compared with

$$
\frac{\zeta(\sigma-\mathrm{i} t)}{\zeta(\sigma)}=\int_{-\infty}^{\infty} e^{\mathrm{i} t y} \mu_{\sigma}^{-}(d y), \quad \mu_{\sigma}^{-}(d y):=\frac{1}{\zeta(\sigma)} \sum_{n=1}^{\infty} \frac{1}{n^{\sigma}} \delta_{\log n}(d y)
$$


proved by (1.2) (see also the beginning of Section 2). Figures 1 and 2 are the graphs of $\left\{P_{2}(y): 0 \leq y \leq 3\right\}$ and $\left\{P_{1 / 2}(y):-1 \leq y \leq 4\right\}$. All figures in this paper are plotted by Mathematica 8.0.

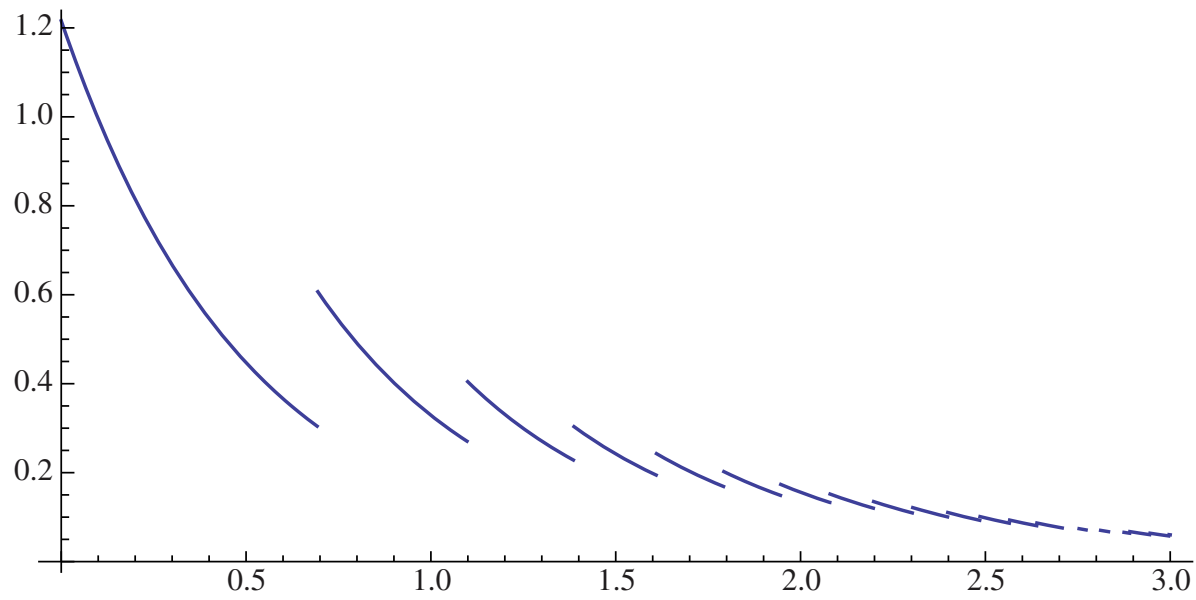

Figure 1. $\left\{P_{2}(y): 0 \leq y \leq 3\right\}$

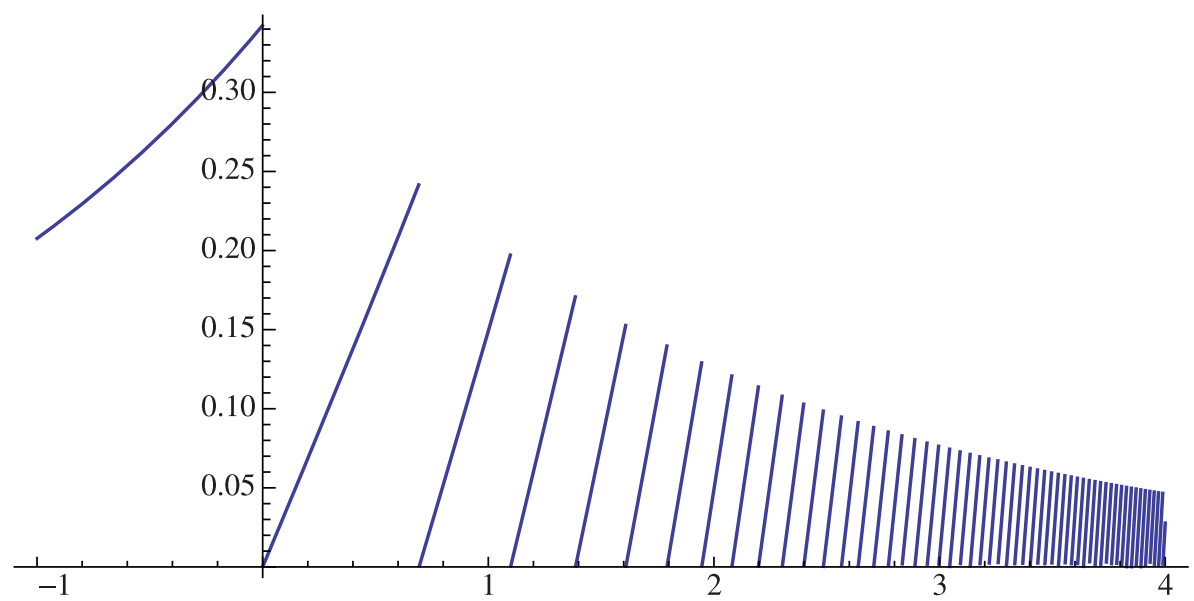

Figure 2. $\left\{P_{1 / 2}(y):-1 \leq y \leq 4\right\}$

Next we consider an application of Theorem 1.2 to analytic number theory.

Theorem 1.3. Let $C \in \mathbb{C}$ satisfy $|C|>10$ and $-19 / 2 \leq \Re C \leq 17 / 2$. Then the function $\zeta(s)+C s$ does not vanish in the half-plane $\sigma>1 / 18$.

We have to mention that the function $\zeta(s)+c$, where $0 \neq c \in \mathbb{C}$ has zeros in the strip $1 / 2<\sigma<1$ (see for example [18, Theorem 1.5]). Moreover, we have the following by [16, Main Theorem 1]. Let $D(s):=\sum_{n=1}^{\infty} a_{n} e^{-\lambda_{n} s}$, where $a_{n} \in \mathbb{C}$ and $\lambda_{1}<\lambda_{2}<\cdots$. Then $\zeta(s)+D(s)$ has zeros in $1 / 2<\sigma<1$ for any $D(s)$ satisfies that $a_{n} \neq 0$ for some $n \in \mathbb{N}$ and the series expression of $D(s)$ converges absolutely 
in $\Re(s)>1 / 2$. Applying this result, we can see that $\zeta(s+1 / 2)-\zeta(s-1 / 2)$ has zeros in the vertical strip $1<\sigma<3 / 2$.

On the contrary, Taylor [19] showed that

$$
\zeta^{*}(s+1 / 2)-\zeta^{*}(s-1 / 2), \quad \zeta^{*}(s):=\pi^{-s / 2} \Gamma(s / 2) \zeta(s)
$$

has all its zeros on the critical line $\sigma=1 / 2$. Hence Theorem 1.3 may be regarded as an analogy of Taylor's result. It is due to the novelty of Theorem 1.3 that we can construct zeta functions that do not have any zero in some strips without the Gamma function $\Gamma(s)$ (see also Hejhal [7] or Lagarias and Suzuki [12] they construct zeta functions having all their zeros on $\sigma=1 / 2$ by using the Gamma function).

\section{ProOFS}

We give a proof of (1.2) to compare with the one of (1.5). Recall that we have $e^{\mathrm{i} x z}=\int_{-\infty}^{\infty} e^{\mathrm{i} z y} \delta_{x} d y$, where $\delta_{x}$ is the delta measure at $x \in \mathbb{R}$. The Riemann zeta function $\zeta(s)$ is written by the Dirichlet series in (1.1) when $\sigma>1$. Hence one has

$$
Z_{\sigma}(t)=\frac{1}{\zeta(\sigma)} \sum_{n=1}^{\infty} \frac{e^{-i t \log n}}{n^{\sigma}}=\frac{1}{\zeta(\sigma)} \sum_{n=1}^{\infty} \frac{1}{n^{\sigma}} \int_{-\infty}^{\infty} e^{\mathrm{i} t y} \delta_{-\log n} d y=\int_{-\infty}^{\infty} e^{\mathrm{i} t y} \mu_{\sigma}(d y)
$$

where $\mu_{\sigma}(d y)$ is defined in (1.2). This equality implies (1.2). Note that we have

$$
\frac{\zeta(\sigma-\mathrm{i} t)}{\zeta(\sigma)}=Z_{\sigma}(-t)=\frac{1}{\zeta(\sigma)} \sum_{n=1}^{\infty} \frac{1}{n^{\sigma}} \int_{-\infty}^{\infty} e^{-\mathrm{i} t z} \delta_{-\log n} d z=\int_{-\infty}^{\infty} e^{\mathrm{i} t y} \mu_{\sigma}^{-}(d y),
$$

where $\mu_{\sigma}^{-}(d y)$ is given in (1.6) by the change of variables integration $z=-y$.

In order to prove Theorem [1.2, we quote the following fact. Let $[x]$ denote the greatest integer not exceeding $x$.

Lemma 2.1 (See [2, p. 246] and [20, (2.1.5)]). It holds that

$$
\begin{aligned}
\zeta(s) & =s \int_{1}^{\infty} \frac{[x]}{x^{s+1}} d x, & & \sigma>1, \\
\zeta(s) & =s \int_{0}^{\infty} \frac{[x]-x}{x^{s+1}} d x, & & 0<\sigma<1 .
\end{aligned}
$$

Proof of Theorem 1.2. First suppose $\sigma>1$. The equation 2.1) is also written $\zeta(s) / s=\int_{0}^{\infty}[x] x^{-s-1} d x$ since we have $[x]=0$ when $0 \leq x<1$. By the change of variables integration $x=e^{y}$, we have

$$
\begin{aligned}
F_{\sigma}(t) & =\frac{\sigma}{\zeta(\sigma)} \int_{0}^{\infty} \frac{[x]}{x^{\sigma-i t+1}} d x=\frac{\sigma}{\zeta(\sigma)} \int_{-\infty}^{\infty} \frac{\left[e^{y}\right]}{e^{y(\sigma-\mathrm{i} t+1)}} e^{y} d y \\
& =\frac{\sigma}{\zeta(\sigma)} \int_{-\infty}^{\infty} \frac{\left[e^{y}\right]}{e^{y(\sigma-\mathrm{i} t)}} d y=\int_{-\infty}^{\infty} e^{\mathrm{i} y t} P_{\sigma}(y) d y
\end{aligned}
$$

where $P_{\sigma}(y)$ is given for $\sigma>1$ by (1.5). Note that $P_{\sigma}(y)=0$ for any $\sigma>1$ and $y<0$ since we have $\left[e^{y}\right]=0$ when $y<0$. One has $\left[e^{y}\right] \geq 0$ for any $y \in \mathbb{R}$ and $\zeta(\sigma)>0$ by the series expression of $\zeta(s)$. Thus it holds that $P_{\sigma}(y) \geq 0$ for any $y \in \mathbb{R}$. Moreover, we have $\int_{-\infty}^{\infty} P_{\sigma}(y) d y=1$ by (2.1). Therefore $P_{\sigma}(y)$ is a probability density function. 
Next suppose $0<\sigma<1$. By the change of variables integration $x=e^{y}$, one has

$$
\begin{aligned}
F_{\sigma}(t) & =\frac{\sigma}{\zeta(\sigma)} \int_{0}^{\infty} \frac{[x]-x}{x^{\sigma-\mathrm{i} t+1}} d x=\frac{\sigma}{\zeta(\sigma)} \int_{-\infty}^{\infty} \frac{\left[e^{y}\right]-e^{y}}{e^{y(\sigma-\mathrm{i} t+1)}} e^{y} d y \\
& =\frac{\sigma}{\zeta(\sigma)} \int_{-\infty}^{\infty} \frac{\left[e^{y}\right]-e^{y}}{e^{y(\sigma-\mathrm{i} t)}} d y=\int_{-\infty}^{\infty} e^{\mathrm{i} y t} P_{\sigma}(y) d y
\end{aligned}
$$

where $P_{\sigma}(y)$ is given for $0<\sigma<1$ by (1.5). We have $\zeta(\sigma) / \sigma<0$ for any $0<\sigma<1$ by (2.2) and $[x]-x \leq 0$ for any $0 \leq x$. Thus it holds that $P_{\sigma}(y) \geq 0$ for any $y \in \mathbb{R}$ since one has $\left[e^{y}\right]-e^{y} \leq 0$ and $\zeta(\sigma) / \sigma<0$. Moreover, we have $\int_{-\infty}^{\infty} P_{\sigma}(y) d y=1$ by (2.2). Thus $P_{\sigma}(y)$ is a probability density function.

Next we prove Theorem 1.3. It is well known that the absolute value of characteristic function is not greater than 1 (see for example [17. Proposition 2.5]). Therefore we immediately have the following inequality by Theorem 1.2

Corollary 2.2. For any $t \in \mathbb{R}$ and $0<\sigma \neq 1$, we have

$$
|\zeta(\sigma+\mathrm{i} t)| \leq \frac{|\zeta(\sigma)|}{\sigma}|\sigma+\mathrm{i} t| .
$$

Let $0<\theta_{0}<\theta_{1}<\theta_{2}$ and put $M:=\max _{\sigma \in\left[\theta_{0}, \theta_{1}\right] \cup\left\{\theta_{2}\right\}}|\zeta(\sigma)| / \sigma$.

Lemma 2.3. For any $C \in \mathbb{C}$ satisfying $|C|>M$, the function $\zeta(s)+C$ s does not vanish in the strips $\theta_{0} \leq \sigma \leq \theta_{1}$ and $\theta_{2} \leq \sigma$.

Proof. Suppose $\sigma>1$. Then $x^{-\sigma-1}, x>1$ is monotonically decreasing. Hence $\zeta(\sigma) / \sigma$ is monotonically decreasing when $\sigma>1$ by (2.1). Thus we have

$$
M:=\max _{\sigma \in\left[\theta_{0}, \theta_{1}\right] \cup\left\{\theta_{2}\right\}}|\zeta(\sigma)| / \sigma=\max _{\sigma \in\left[\theta_{0}, \theta_{1}\right] \cup\left[\theta_{2}, \infty\right)}|\zeta(\sigma)| / \sigma .
$$

By using (2.3) and the equation above, we have

$$
|\zeta(s)| \leq M|s|, \quad \sigma \in\left[\theta_{0}, \theta_{1}\right] \cup\left[\theta_{2}, \infty\right) .
$$

Hence $\zeta(s)+C s$ with $|C|>M$ does not vanish in the strips $\theta_{0} \leq \sigma \leq \theta_{1}$ and $\theta_{2} \leq \sigma$.

As mentioned above, $\zeta(\sigma) / \sigma$ is monotonically decreasing if $\sigma>1$. On the other hand, for $0<\sigma<1$, we have

$$
\frac{d^{2}}{d^{2} \sigma} \frac{|\zeta(\sigma)|}{\sigma}=\frac{d^{2}}{d^{2} \sigma} \int_{0}^{\infty} \frac{x-[x]}{x^{\sigma+1}} d x=\int_{0}^{\infty} \frac{(x-[x]) \log ^{2} x}{x^{\sigma+1}}>0
$$

by (2.2). Moreover, Mathematica 8.0 gives Figure 3 and the following numerical values:

$$
\begin{aligned}
\left|(1 / 18)^{-1} \zeta(1 / 18)\right| & =9.97794103359879215955145424246 \ldots \\
\left|(8 / 9)^{-1} \zeta(8 / 9)\right| & =9.48480110687167088364420788938 \ldots \\
\left|(11 / 10)^{-1} \zeta(11 / 10)\right| & =9.62222587722800893307854617431 \ldots .
\end{aligned}
$$

Hence Figure 3 indicates that we can take $M=10$ when $1 / 18 \leq \theta_{0}<\theta_{1} \leq 8 / 9$ and $11 / 10 \leq \theta_{2}$ in Lemma 2.3 . Therefore $\zeta(s)+C s$ with $|C|>10$ does not vanish in the strips $1 / 18 \leq \sigma \leq 8 / 9$ and $11 / 10 \leq \sigma$. Thus we only have to show the following lemma.

Lemma 2.4. Let $C \in \mathbb{C}$ satisfy $|C|>10$ and $-19 / 2 \leq \Re C \leq 17 / 2$. Then the function $\zeta(s)+C$ s does not vanish in the strip $8 / 9<\sigma<11 / 10$. 


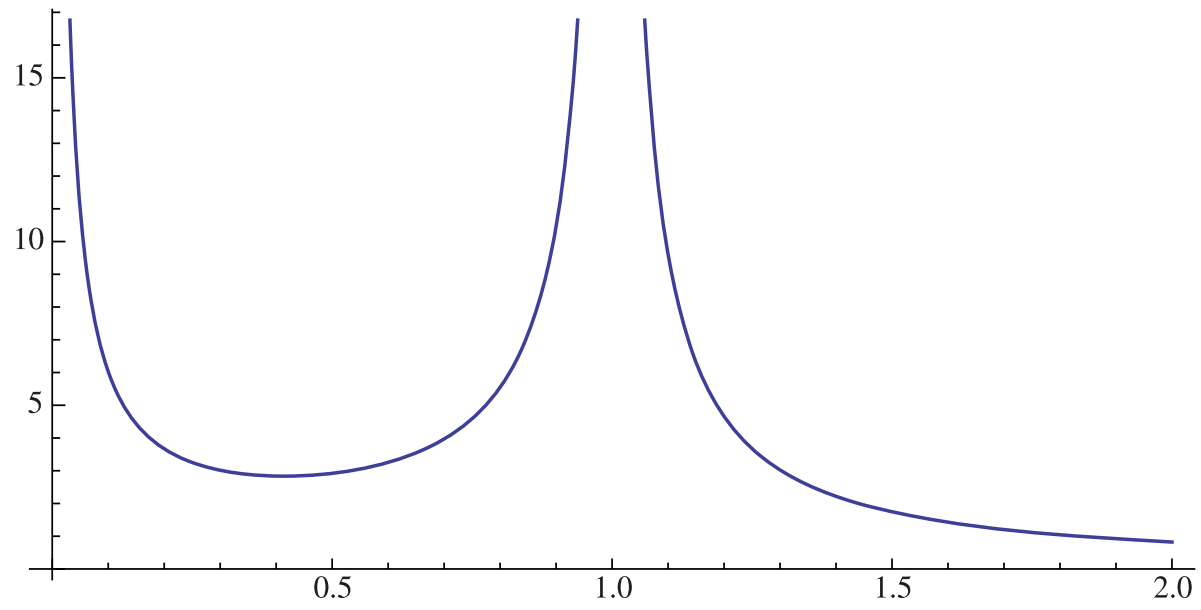

Figure 3. $\{|\zeta(\sigma)| / \sigma: 0<\sigma<2\}$

Proof. For $\sigma>0$, it is known (see [8, (1.7)]) that

$$
\zeta(s)=\frac{s}{s-1}+\operatorname{sh}(s), \quad h(s):=\int_{1}^{\infty} \frac{[x]-x}{x^{s+1}} d x .
$$

By $[x]-x \leq 0$ for any $x \geq 1$ and the integral representation of $h(s)$, we have

$$
|h(s)| \leq \int_{1}^{\infty} \frac{|[x]-x|}{\left|x^{s+1}\right|} d x=\int_{1}^{\infty} \frac{x-[x]}{x^{\sigma+1}} d x=-h(\sigma) .
$$

Therefore it holds that

$$
\begin{aligned}
\sup _{8 / 9<\sigma<11 / 10}|h(s)| & =\sup _{8 / 9<\sigma<11 / 10}\left\{\int_{1}^{\infty} \frac{x-[x]}{x^{\sigma+1}} d x\right\}=-h(8 / 9)=\frac{1}{8 / 9-1}-\frac{\zeta(8 / 9)}{8 / 9} \\
& =0.484801106871670883644207889377 \ldots<1 / 2 .
\end{aligned}
$$

First suppose $|s-1| \geq 1$. In this case, one has $\left|s(s-1)^{-1}\right| \leq|s|$. On the other hand, we have $1<|h(s)+C|$ by (2.4) and the assumption $|C|>10$. Therefore

$$
\zeta(s)+C s=\frac{s}{s-1}+(h(s)+C) s
$$

does not vanish when $8 / 9<\sigma<11 / 10$ and $\left|(s-1)^{-1}\right| \leq 1$.

Next suppose $|s-1|<1$. Obviously, $\zeta(s)+C s=0$ is equivalent to

$$
s=1-\frac{1}{h(s)+C}
$$

by (2.5). Thus $\zeta(s)+C s$ does not vanish in the vertical strip $8 / 9<\sigma<11 / 10$ if

$$
1-\Re \frac{1}{h(s)+C} \leq \frac{8}{9} \quad \text { or } \quad \frac{11}{10} \leq 1-\Re \frac{1}{h(s)+C} .
$$

By an easy computation, we can see that the condition $-10 \leq \Re h(s)+C \leq 9$ satisfies (2.6). On the other hand, it holds that $|\Re h(s)|<1 / 2$ by (2.4). Hence $-19 / 2 \leq \Re C \leq 17 / 2$ fulfills the condition (2.6). 
Remark 2.5. From [8, p. 9], we have $\zeta(s)=\chi(s) \zeta(1-s)$ and

$$
\chi(s):=\frac{(2 \pi)^{s}}{2 \Gamma(s) \cos (\pi s / 2)}=(2 \pi / t)^{\sigma+\mathrm{i} t-1 / 2} e^{\mathrm{i}(t+\pi / 4)}\left(1+O\left(t^{-1}\right)\right), \quad t \geq t_{0}>0 .
$$

Therefore for any $C_{+}>0$, there exists $t>0$ such that $|\zeta(\sigma+\mathrm{i} t)|>C_{+}|\sigma+\mathrm{i} t|$ when $\sigma<-1 / 2$. Hence $\zeta(s)+C s$ has zeros for some suitable $C$ when $\sigma<-1 / 2$. For example, we have

$$
\begin{aligned}
& C_{1}^{\prime}:=\frac{\zeta\left(-1+\mathrm{i} 10^{10}\right)}{-1+\mathrm{i} 10^{10}}=5322.98794190618 \ldots-\mathrm{i} 519.09996008851 \ldots, \\
& C_{2}^{\prime}:=\frac{\zeta\left(-2+\mathrm{i} 10^{10}\right)}{-2+\mathrm{i} 10^{10}}=9.51272107949384 \ldots \times 10^{12}-\mathrm{i} 3.79619848933398 \ldots \times 10^{12} .
\end{aligned}
$$

Thus one has $\zeta(s)-C_{1}^{\prime} s=0$ when $s=-1+\mathrm{i} 10^{10}$ and $\zeta(s)-C_{2}^{\prime} s=0$ when $s=-2+\mathrm{i} 10^{10}$ by the definitions of $C_{1}^{\prime}$ and $C_{2}^{\prime}$. These should be compared with the fact that $\zeta(s)+C s$ with $|C|>10$ does not vanish in the strips $1 / 18 \leq \sigma \leq 8 / 9$ and $11 / 10 \leq \sigma$ (see the sentences above Lemma 2.4).

\section{Some ReMARKS FROM THE VIEW OF INFINITE DIVISIBILITY}

A probability measure $\mu$ on $\mathbb{R}$ is infinitely divisible if, for any positive integer $n$, there is a probability measure $\mu_{n}$ on $\mathbb{R}$ such that $\mu=\mu_{n}^{n *}$, where $\mu_{n}^{n *}$ is the $n$-fold convolution of $\mu_{n}$. For example, normal, degenerate, Poisson and compound Poisson distributions are infinitely divisible. Infinitely divisible distributions are the marginal distributions of stochastic processes having independent and stationary increments such as Brownian motion and Poisson processes. Such stochastic processes were well studied by P. Lévy and now we usually call them Lévy processes (see for example [17]).

Let $\widehat{\mu}(t)$ be the characteristic function of a probability measure $\mu$ on $\mathbb{R}$ and $I D(\mathbb{R})$ be the class of all infinitely divisible distributions on $\mathbb{R}$. The following Lévy-Khintchine representation is well known (see [17, Section 2]). If $\mu \in I D(\mathbb{R})$, then we have

$$
\widehat{\mu}(t)=\exp \left[-\frac{a}{2} t^{2}+\mathrm{i} \gamma t+\int_{\mathbb{R}}\left(e^{\mathrm{i} t x}-1-\frac{\mathrm{i} t x}{1+|x|^{2}}\right) \nu(d x)\right], \quad t \in \mathbb{R},
$$

where $a \geq 0, \gamma \in \mathbb{R}$ and $\nu$ is a measure on $\mathbb{R}$ that satisfies $\nu(\{0\})=0$ and $\int_{\mathbb{R}}\left(|x|^{2} \wedge 1\right) \nu(d x)<\infty$. Moreover, the representation of $\widehat{\mu}$ in (3.1) by $a, \nu$, and $\gamma$ is unique. Note that if the Lévy measure $\nu$ in (3.1) satisfies $\int_{|x|<1}|x| \nu(d x)<\infty$, then (3.1) can be written by

$$
\widehat{\mu}(t)=\exp \left[-\frac{a}{2} t^{2}+\mathrm{i} \gamma_{0} t+\int_{\mathbb{R}}\left(e^{\mathrm{i} t x}-1\right) \nu(d x)\right], \quad t \in \mathbb{R},
$$

where $\gamma_{0}=\gamma-\int_{\mathbb{R}} x\left(1+|x|^{2}\right)^{-1} \nu(d x)$.

Let $\widehat{I D}(\mathbb{R})$ be the set of all infinitely divisible characteristic functions on $\mathbb{R}$. We can see that $Z_{\sigma}(t):=\zeta(\sigma+\mathrm{i} t) / \zeta(\sigma) \in \widehat{I D}(\mathbb{R})$ from (1.3) and (3.2). As an analogy of this fact, we have the following theorem by Proposition 1.1 . 
Theorem 3.1. When $\sigma>1$, we have

$$
\begin{aligned}
& F_{\sigma}(t)=\exp \left[\int_{0}^{\infty}\left(e^{\mathrm{i} t x}-1\right) N_{\sigma}^{*}(d x)\right], \\
& N_{\sigma}^{*}(d x):=x^{-1} e^{-\sigma x}(d x)+\sum_{p} \sum_{r=1}^{\infty} \frac{p^{-r \sigma}}{r} \delta_{r \log p}(d x) .
\end{aligned}
$$

Especially, the function $F_{\sigma}(t) \in \widehat{I D}(\mathbb{R})$ is a compound Poisson characteristic function when $\sigma>1$.

Proof. We can find the Lévy measure of $\sigma /(\sigma-\mathrm{i} t)$ by

$$
\frac{\sigma}{\sigma-\mathrm{i} t}=\exp \left[\int_{0}^{\infty}\left(e^{\mathrm{i} t x}-1\right) x^{-1} e^{-\sigma x} d x\right]
$$

(see for instance [17, p. 45]). Hence one has $\sigma /(\sigma-\mathrm{i} t) \in \widehat{I D}(\mathbb{R})$ by $(3.2)$. On the other hand, it holds that

$$
F_{\sigma}(t)=\frac{\zeta(\sigma-\mathrm{i} t)}{\zeta(\sigma)} \frac{\sigma}{\sigma-\mathrm{i} t}
$$

by the definition of $F_{\sigma}(t)$. Therefore we obtain Theorem 3.1 by (1.3).

The infinite divisibility of $F_{\sigma}(t)$ for $1 / 2<\sigma<1$ is very interesting since we have the following theorem related to the Riemann hypothesis which states that $\zeta(s) \neq 0$ when $\sigma>1 / 2$.

Theorem 3.2. The Riemann hypothesis is true if $F_{\sigma}(t) \in \widehat{I D}(\mathbb{R})$ for any $1 / 2<$ $\sigma<1$.

Proof. Note that $\zeta(1+\mathrm{i} t) \neq 0$ for any $t \neq 0$ (see for example [2, Theorem 13.6]). On the other hand, it is known that $\widehat{\mu}(t) \neq 0$ for any $t \in \mathbb{R}$ if $\widehat{\mu}(t) \in \widehat{I D}(\mathbb{R})$ (see [17. Lemma 7.5]). Hence $\zeta(\sigma+\mathrm{i} t) \neq 0$ if $F_{\sigma}(t) \in \widehat{I D}(\mathbb{R})$.

Remark 3.3. We cannot expect that the converse of Theorem 3.2 is true by the value-distribution theory of $\zeta(s)$ (see the sentences below Theorem 1.3). Note that there are many papers and books on value-distribution theory of zeta-functions in probabilistic view; for example, [3, [9, 13, [15] and [18.

However, if we consider

$$
F_{\sigma}(t, n):=\frac{\sigma^{n}}{(\sigma-\mathrm{i} t)^{n}} \frac{\zeta(\sigma-\mathrm{i} t)}{\zeta(\sigma)}=\frac{\sigma^{n-1}}{(\sigma-\mathrm{i} t)^{n-1}} F_{\sigma}(t),
$$

for sufficiently large $n \in \mathbb{N}$, then $F_{\sigma}(t, n)$ might be an infinitely divisible characteristic function under the Riemann hypothesis. This is explained as follows. For $0<\sigma<1, F_{\sigma}(t, n)$ is a characteristic function since $\sigma /(\sigma-\mathrm{i} t)$ and $F_{\sigma}(t)$ are characteristic functions, and it is known that the product of a finite number of characteristic functions is also a characteristic function. By (3.3), we have $\sigma^{n}(\sigma-\mathrm{i} t)^{-n} \in \widehat{I D}(\mathbb{R})$ for any $n \in \mathbb{N}$. Then we can guess that the effect of $F_{\sigma}(t)$ in the function $F_{\sigma}(t, n)$ might be small when $n \in \mathbb{N}$ is sufficiently large (recall Corollary 2.2). In other words, if we could consider a function $G_{\sigma}(t) F_{\sigma}(t)$, where $G_{\sigma}(t)$ is a 'suitable' infinitely divisible characteristic function, then $G_{\sigma}(t) F_{\sigma}(t)$ would be an infinitely divisible characteristic function under the Riemann hypothesis. 


\section{REFERENCES}

[1] T. Aoyama and T. Nakamura, Multidimensional polynomial Euler products and infinitely divisible distributions on $\mathbb{R}^{d}$, submitted, (2012), arxiv.org/abs/1204.4041.

[2] Tom M. Apostol, Introduction to analytic number theory, Springer-Verlag, New York, 1976. Undergraduate Texts in Mathematics. MR0434929 (55 \#7892)

[3] Harald Bohr and Börge Jessen, Über die Werteverteilung der Riemannschen Zetafunktion (German), Acta Math. 58 (1932), no. 1, 1-55, DOI 10.1007/BF02547773. MR.1555343

[4] Philippe Biane, Jim Pitman, and Marc Yor, Probability laws related to the Jacobi theta and Riemann zeta functions, and Brownian excursions, Bull. Amer. Math. Soc. (N.S.) 38 (2001), no. 4, 435-465 (electronic), DOI 10.1090/S0273-0979-01-00912-0. MR1848256 (2003b:11083)

[5] B. V. Gnedenko and A. N. Kolmogorov, Limit distributions for sums of independent random variables, Translated from the Russian, annotated, and revised by K. L. Chung. With appendices by J. L. Doob and P. L. Hsu. Revised edition, Addison-Wesley Publishing Co., Reading, Mass.-London-Don Mills., Ont., 1968. MR0233400 (38 \#1722)

[6] Allan Gut, Some remarks on the Riemann zeta distribution, Rev. Roumaine Math. Pures Appl. 51 (2006), no. 2, 205-217. MR2275304(2007m:11117)

[7] Dennis A. Hejhal, On a result of G. Pólya concerning the Riemann $\xi$-function, J. Analyse Math. 55 (1990), 59-95, DOI 10.1007/BF02789198. MR1094712 (92c:11088)

[8] Aleksandar Ivić, The Riemann zeta-function, Dover Publications Inc., Mineola, NY, 2003. Theory and applications; Reprint of the 1985 original [Wiley, New York; MR0792089 (87d:11062)]. MR 1994094

[9] Børge Jessen and Aurel Wintner, Distribution functions and the Riemann zeta function, Trans. Amer. Math. Soc. 38 (1935), no. 1, 48-88, DOI 10.2307/1989728. MR 1501802

[10] A. Ya. Khintchine, Limits Theorem for Sums of Independent Random Variables (in Russian), Moscow and Leningrad, 1938.

[11] J. C. Lagarias and E. Rains, On a two-variable zeta function for number fields (English, with English and French summaries), Ann. Inst. Fourier (Grenoble) 53 (2003), no. 1, 1-68. MR.1973068 (2004f:11096)

[12] Jeffrey C. Lagarias and Masatoshi Suzuki, The Riemann hypothesis for certain integrals of Eisenstein series, J. Number Theory 118 (2006), no. 1, 98-122, DOI 10.1016/j.jnt.2005.08.010. MR2220265(2007c:11099)

[13] Antanas Laurinčikas, Limit theorems for the Riemann zeta-function, Mathematics and its Applications, vol. 352, Kluwer Academic Publishers Group, Dordrecht, 1996. MR 1376140 (96m:11070)

[14] Gwo Dong Lin and Chin-Yuan Hu, The Riemann zeta distribution, Bernoulli 7 (2001), no. 5, 817-828, DOI 10.2307/3318543. MR1867083(2003k:11134)

[15] Kohji Matsumoto, Probabilistic value-distribution theory of zeta-functions [translation of Sūgaku 53 (2001), no. 3, 279-296; MR1850006], Sugaku Expositions 17 (2004), no. 1, 51-71. Sugaku Expositions. MR2073363

[16] T. Nakamura and Łukasz Pańkowski, On complex zeros off the critical line for non-monomial polynomial of zeta-functions, submitted, (2012), arXiv:1212.5890.

[17] Ken-iti Sato, Lévy processes and infinitely divisible distributions, Cambridge Studies in Advanced Mathematics, vol. 68, Cambridge University Press, Cambridge, 1999. Translated from the 1990 Japanese original; Revised by the author. MR 1739520 (2003b:60064)

[18] Jörn Steuding, Value-distribution of L-functions, Lecture Notes in Mathematics, vol. 1877, Springer, Berlin, 2007. MR2330696 (2008m:11172)

[19] P. R. Taylor, On the Riemann zeta function, Quart. J. Math., Oxford Ser. 16 (1945), 1-21. $\operatorname{MR} 0012626(7,48 \mathrm{~g})$

[20] E. C. Titchmarsh, The theory of the Riemann zeta-function, 2nd ed., The Clarendon Press Oxford University Press, New York, 1986. Edited and with a preface by D. R. Heath-Brown. MR:882550(88c:11049)

Graduate School of Mathematical Sciences, The University of Tokyo 3-8-1 Komaba

MEguro-Ku TOKYO 153-8914, JAPAN

E-mail address: takashin@ms.u-tokyo.ac.jp 